\title{
PSYCHOSOCIAL FACTORS INFLUENCE CONTROL OF MODERATE AND SEVERE HYPERTENSION
}

\author{
John R. Caldwell ${ }^{1.4}$, Velma Theisen ${ }^{1}$, Catherine A. Kaunisto ${ }^{2}$, Patrick J. Reddy ${ }^{3}$, \\ Peter S. SMYTHE ${ }^{4}$ and DAVId W. SMith ${ }^{1}$ \\ ${ }^{1}$ Hypertension Clinic, Henry Ford Hospital, Detroit MI 48202, ${ }^{2}$ D. J. Sullivan \& Associates. Ann Arbor, MI, \\ ${ }^{3}$ St Louis University School of Medicine, St Louis, MO 63103 and ${ }^{4}$ Department of Internal Medicine, \\ University of Michigan Medical School, Ann Arbor, MI 48109, U.S.A.
}

\begin{abstract}
Factors affecting the degree of blood pressure control achieved by antihypertensive drug treatment in 150 patients with moderate and severe essential hypertension were analyzed. Patients were under continuing treatment for 1-23 years. Most patients were referred for hypertension which was difficult to control. All were managed by the senior author. They received multiple drug regimens and extensive efforts were made to encourage their adherence to the regimens. In spite of these efforts, 16 $(11 \%)$ had poor blood pressure control. Satisfactory control was achieved in $52(35 \%)$ patients and excellent control in $82(55 \%)$ patients. As a group, their ideal body weight (IBW) was $125 \pm 22 \%$ (mean \pm SD) of normal. The mean number of antihypertensive tablets per day was significantly greater $(P=0.0009)$ in those with poor control as compared to those with satisfactory and excellent control. As $\%$ IBW increased, the number of antihypertensive tablets increased $(P=0.0021)$. We examined the relationship of blood pressure control with an index of compliance, with psychosocial factors (life events score, marital status) and with socioeconomic factors (work status, income). Poor blood pressure control was associated with a lower compliance index $(P<0.0001)$ and a higher life events score $(P<0.006)$. Poorly controlled patients were more likely to have poor health in general $(P=0.0002)$, to have kept fewer medical appointments during the preceding year $(P<0.0001)$, to be unmarried $(P<0.0001)$, to be unemployed $(P<0.0028)$ and have a lower income $(P<0.009)$. As \% IBW increased, compliance index decreased. $(P=0.0045)$. Therefore, psychosocial and socioeconomic factors, as well as physical factors, influence blood pressure control in moderate and severe essential hypertension.
\end{abstract}

Blood pressure control reduces mortality in mild, moderate or severe hypertension [1]. Also, an improvement in blood pressure control has been noted during the last decade. For example, more than $50 \%$ of stepped care patients in the Hypertension Detection and Follow-up Programs achicved blood pressure levels within the normotensive range or at or below the goal for diastolic blood pressure. This represents considerable improvement over the estimate of $29^{\circ}$ of hypertensive patients under good control in 1974 [2]. In Chicago in $1977.73 \%$ of hypertensives had adequately controlled blood pressure as contrasted to $59 \%$ in the previous year and $21 \%$ in 1972 [3]. However despite the striking advances in antihypertensive treatment. at least ! in 5 treated hypertensive patients is inadequately controlled [4]. Probably the most frequently cited reason for inadequate control is poor patient compliance with antihypertensive treatment [5-7].

Patients may fail to comply with treatment in many ways. The most severe degree of noncompliance is complete withdrawal from treatment. We have had clinical experience with the disastrous consequences of this behavior [8]. We have also been concerned with lesser degrees of noncompliance which contrib-

Portions of this report were originally presented by $\mathrm{Dr}$ John R. Caldwell at the National Conference on High Blood Pressure Control. Houston. TX. 25 March 1980 and at the American Psychosomatic Society Annual Meeting. New York. 1980 ute to poor blood pressure control. While the obvious problem is not taking medication as prescribed, noncompliance may also be manifested by missing scheduled appointments and by disregarding dietary instructions (both caloric and low sodium intake). More subtle noncompliance includes ignorance of the drug regimen, and failure to recognize the importance of taking medications, not keeping appointments and not refilling prescriptions on time.

In our practice, we have tried to achieve a goal blood pressure below $140 \mathrm{~mm} \mathrm{Hg}$ systolic and $90 \mathrm{~mm} \mathrm{Hg}$ diastolic whenever possible. However, we have been frustrated in achieving and maintaining this goal in some patients with moderate or severe essential hypertension. Others have reported that even some patients being treated with minoxidil for resistant hypertension are inadequately controlled due to noncompliance [9]. We posed this question: Why are some intensively treated hypertensive patients inadequately controlled? To answer this question, we evaluated psychosocial factors and compliance using an interview, a questionnaire, and a chart review. We excluded obvious secondary hypertension which might be a cause of resistance to treatment.

\section{METHODS}

One hundred and fifty patients under continuing treatment by the senior author for moderate and severe essential hypertension were studied. All 
Table 1. Descriptive characteristics for 150 patients with essential hypertension, age 30-75, at time of study

\begin{tabular}{|c|c|}
\hline Characteristic & \\
\hline Age (years) $( \pm S D)^{*}$ & $59 \pm 10$ \\
\hline Male & $89(59 \%)$ \\
\hline Female & $61(41 \%)$ \\
\hline White & $84(56 \%)$ \\
\hline Black & $64(43 \%)$ \\
\hline Other race & $2(1 \%)$ \\
\hline $\begin{array}{l}\text { Duration of prior treatment (years) } \\
( \pm S D)\end{array}$ & $11 \pm 7$ \\
\hline $\begin{array}{l}\text { Systolic blood pressure, initial } \uparrow \\
(\mathrm{mm} \mathrm{Hg})( \pm \mathrm{SD})\end{array}$ & $178 \pm 30$ \\
\hline $\begin{array}{l}\text { Systolic blood pressure, final }+ \\
(\mathrm{mm} \mathrm{Hg})\end{array}$ & $142 \pm 23$ \\
\hline $\begin{array}{l}\text { Diastolic blood pressure, initial } \\
(\mathrm{mm} \mathrm{Hg})( \pm \mathrm{SD})\end{array}$ & $108 \pm 17$ \\
\hline $\begin{array}{l}\text { Diastolic blood pressure. final } \\
(\mathrm{mm} \mathrm{Hg})( \pm \mathrm{SD})\end{array}$ & $84 \pm 12$ \\
\hline $\begin{array}{l}\text { Number antihypertensive tablets per day } \\
( \pm \text { SD })\end{array}$ & $6 \pm 3$ \\
\hline Weight (pounds) ( $\pm S D)$ & $180 \pm 38$ \\
\hline Pounds above ideal body weight & $35 \pm 32$ \\
\hline$\%$ of ideal body weight $( \pm S D)$ & $125 \pm 22$ \\
\hline
\end{tabular}

${ }^{*} \pm \mathrm{SD}=$ standard deviation.

†Initial = at entry to Hypertension Clinic.

${ }_{+}$Final $=$most recent office visit.

patients had been treated for at least 1 year and were taking at least 2 or more antihypertensive medications. Patients were between 30 and 75 years old, averaging 59 years. The duration of treatment was 1-23 years, averaging 11 years. $59 \%$ of the patients were male and $56 \%$ were white. Characteristics of the patients studied are shown in Table 1.

Target organ damage was assessed by chest roentgenogram, electrocardiogram, serum creatinine, urinalysis and funduscopic examination. By chart review, we determined previous complications of hypertension and associated athersclerotic arterial disease. A few of the commonly associated medical problems observed in these patients were also recorded (Table 2). Secondary hypertension was excluded by history, physical examination, the usual laboratory tests and, where indicated, by a rapid sequence intravenous pyelogram, renal arteriogram and renal vein renin study.

Many different individual drug regimens were used. When we classified the regimens according to stepped care [1], the following regimens were used: Step I, a diuretic plus a potassium sparing agent. $2(1 \%)$; Step 2 , a diuretic plus an adrenergic blocker, $64(43 \%)$; Step 3, a diuretic plus an adrenergic blocker, plus a vasodilator, $64(43 \%)$; Step 4, a diuretic plus an adrenergic blocker, plus a vasodilator and/or guanethidine or minoxidil (as a vasodilator), $8(5 \%)$. Other combinations, $12(8 \%)$, were diuretic and guanethidine, diuretic and vasodilator, adrenergic blocker and vasodilator drugs. Combination tablets were used whenever possible to enhance patient compliance. Such combinations were counted as one tablet when we totalled the number of tablets prescribed per day.

Seventy-five patients $(50 \%)$ were seen at scheduled intervals by a clinical nurse specialist, who counseled them on compliance to antihypertensive regimens. achieving goal blood pressures and improving their health education. The majority of the patients had been referred for treatment of difficult hypertension. The care of each patient was managed individually by the senior author on a fee for service basis. (Most had Blue Bross Blue Shield insurance coverage.) The patients were known to receptionists, nurses and nurses aides. as well as to the secretary and the clinical nurse specialist. If an appointment was not kept. a notice of missed appointment was sent to the patient the same day with recommendation for making a new appointment. All patients received comprehensive medical care in addition to hypertension control. A pharmacy served the clinic floor where waiting time was short and individual pharmacist consultation was the rule. We tried to achieve what the stepped care treatment regimen did in the Hypertension Detection and Follow-Up Program. The clinic setting was supportive of adherence to treatment and maximum efforts were made to achieve goal blood pressure with a minimum of side effects. An important difference between our study and the HDFP was that in our study the office visits and prescriptions were not free. and free transportation was never provided.

After a routine office visit, all patients who fulfilled the criteria (treatment at least 1 year. 2 or more different types of antihypertensive drugs prescribed) were asked to participate in an interview. The interviews. conducted by one of two medical students. lasted about $30 \mathrm{~min}$ and included a comprehensive questionnaire. The interviewer also reviewed the medical record of eaçh patient. The physician also reviewed the medical record and completed a questionnaire as well. The questionnaires and medical record review forms were developed by a clinical nurse specialist, in collaboration with the senior author. Further retrospective review of their medical records provided information about the patient's clinical course from the time of entry (initial) into the Hypertension Clinic to their most recent (final) office visit.

Table 2. Cardiovascular renal manifestations, complications and associated problems in 150 patients, age $30-75$. at time of study

\begin{tabular}{lr}
\hline Manifestations & \\
Cardiac enlargement (roentgenogram) & $17\left(11^{\circ}\right)$ \\
Left ventricular hypertrophy (ECG) & $27\left(18^{\circ}\right)$ \\
Azotemia (serum creatinine $>1.3 \mathrm{mg} / \mathrm{dl}$ & $40\left(27^{\circ}\right)$ \\
Proteinuria $(1+$ or $>$ ) & $13\left(9^{\circ}\right)$ \\
Retinopathy (gr. 3 or 4$)$ & $7\left(5^{\circ}\right)$ \\
Complications (by history at chart review) & \\
Stroke & $7\left(5^{\circ} \%\right)$ \\
Myocardial infarction & $11\left(7^{\circ} \%\right)$ \\
Congestive heart failure & $13\left(9^{\circ} \%\right)$ \\
Cardiac arrhythmias or angina & $11\left(7^{\circ} \%\right)$ \\
Peripheral vascular disease & $20\left(13^{\circ} \%\right)$ \\
Renal failure & $9\left(6^{\circ} \%\right)$ \\
Associated problems & \\
Diabetes mellitus & $30\left(20^{\circ}\right)$ \\
treated with diet alone & $14\left(9^{\circ}\right)$ \\
treated with diet and insulin & $10\left(7^{\circ}\right)$ \\
treated with oral hypoglycemics & $6\left(4^{\circ} \%\right)$ \\
Anxiety tension state & $29\left(19^{\circ} \%\right)$ \\
Osteoarthritis & $22\left(15^{\circ}\right)$ \\
Hospitalization $>3$ times for high & \\
blood pressure & $22\left(15^{\circ} \%\right)$ \\
\hline
\end{tabular}


The physician assigned patients to one of three categories of blood pressure control; poor, satisfactory, and excellent. Poor blood pressure control was defined as blood pressure greater than 160 systolic and 90 diastolic at all visits in the past year. Satisfactory control was defined as blood pressure often below 160 systolic or 90 diastolic but not always less than $140 / 90 \mathrm{~mm} \mathrm{Hg}$ in the past year. Isolated (treated) systolic hypertension ( $>160 \mathrm{~mm} \mathrm{Hg}$ ) in older patients was also included in this group if their diastolic was less than $90 \mathrm{~mm} \mathrm{Hg}$. Excellent control was defined as all readings less than 140 systolic and 90 diastolic in the past year.

A compliance index was developed from data collected in the interview-questionnaire. A point score was developed for each of five items (Fig. 1). The questions used in the interview are available from the authors on request. Even an admission of noncompliance with diet or drug regimen was given a point score of 1 so that integers would be entered into the computer for final scoring. Therefore, 5 is subtracted from the original score in rescaling the score to range from 0 (minimum compliance) to 10 (maximum compliance). A histogram of the compliance index for all patients appeared to be normally distributed, although this was not intentional. In order to assess the impact of psychosocial factors on the compliance index, a life events score was derived from a questionnaire listing 12 disturbing life events (Fig. 2). This was a modification of Holmes and Rahe's social readjustment rating scale [10] developed by Harburg (consul$\tan t$ ). In addition to the number of life change units during the preceding 6 months, a measure of the degree of impact on the individual in included.

The percentage of ideal body weight for each individual was determined based on a comparison of actual weight in pounds at most recent office visits and a table of norms. The table of norms was taken from Metropolitan Life Insurance Company data. It was based on one table for desirable weights for men of ages 25 and over (weight in pounds according to medium frame in indoor clothing) and heights (with shoes on, with approx. 1 in. heels). Height was expressed in feet and inches. A similar table was used for women (heights were with shoes on and approx. 2 in. heels).

All of the data were entered on forms suitable for computerized data processing and were analyzed using a variety of statistical procedures, including Student's $t$-tests, chi-square tests, correlation coefficients and analysis of variance (ANOVA).

\section{RESULTS}

\section{Results of antihypertensive treatment}

Blood pressure responses to antihypertensive treatment are shown in Fig. 3. The initial blood pressures were lower than usual for patients with as much target organ damage as our patients (Table 2). This is probably because more than half were already being treated when they entered the Hypertension Clinic (initial blood pressure reading). The final blood pressure measurement for the group as a whole

\section{Compliance Index (C.I.)}

Kept return visits within past year $>75 \%$ of visits ( \pm 30 days)

$$
\begin{aligned}
& 50-75^{\circ} \text { of visits ( } \pm 30 \text { days) } \\
& <50 \% \text { of visits ( } \pm 30 \text { days) }
\end{aligned}
$$$$
\text { Diet }
$$

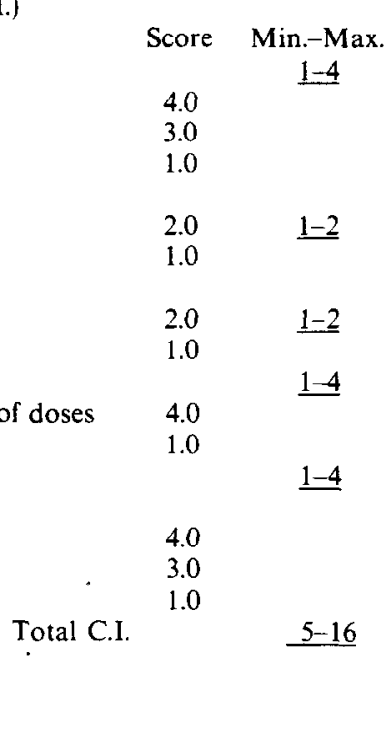

Affirms compliance

Admits noncompliance

Drug regimens

Affirms compliance

Admits noncompliance

Knowledge of drug regimens

Kind. dose. number of tablets per dose, frequency of doses

$<50^{\circ}$ o knowledge (no. items known/total)

Importance of taking medications, keeping

appointments and refilling prescriptions on time

Extremely important all items

Fairly important

Not too important

Rescaled score $=10 / 11$ (Original score) -5

$0 \leqslant$ rescaled score $\leqslant 10$

$2.2 \leqslant$ actual range $\leqslant 9.2$

Fig. 1. The first item was an objective measure obtained from the medical record. The second, third, and fifth items were self-reported and subjective (derived from the questionnaire). Item four was based on comparison between what the patient reported in the questionnaire and the prescribed regimen according to the medical record. A correlation matrix of the five items shows that separate items are not correlated with each other. but that each of these components contribute to the overall compliance index and that all parts. taken collectively. provide a much stronger measure of compliance. The mean compliance index for the 150 patients studied was $6.7 \pm 1.2$ (mean \pm SD). 
Here is a list of life events that happen to many people. Within the past 6 months how many of these did you experience?

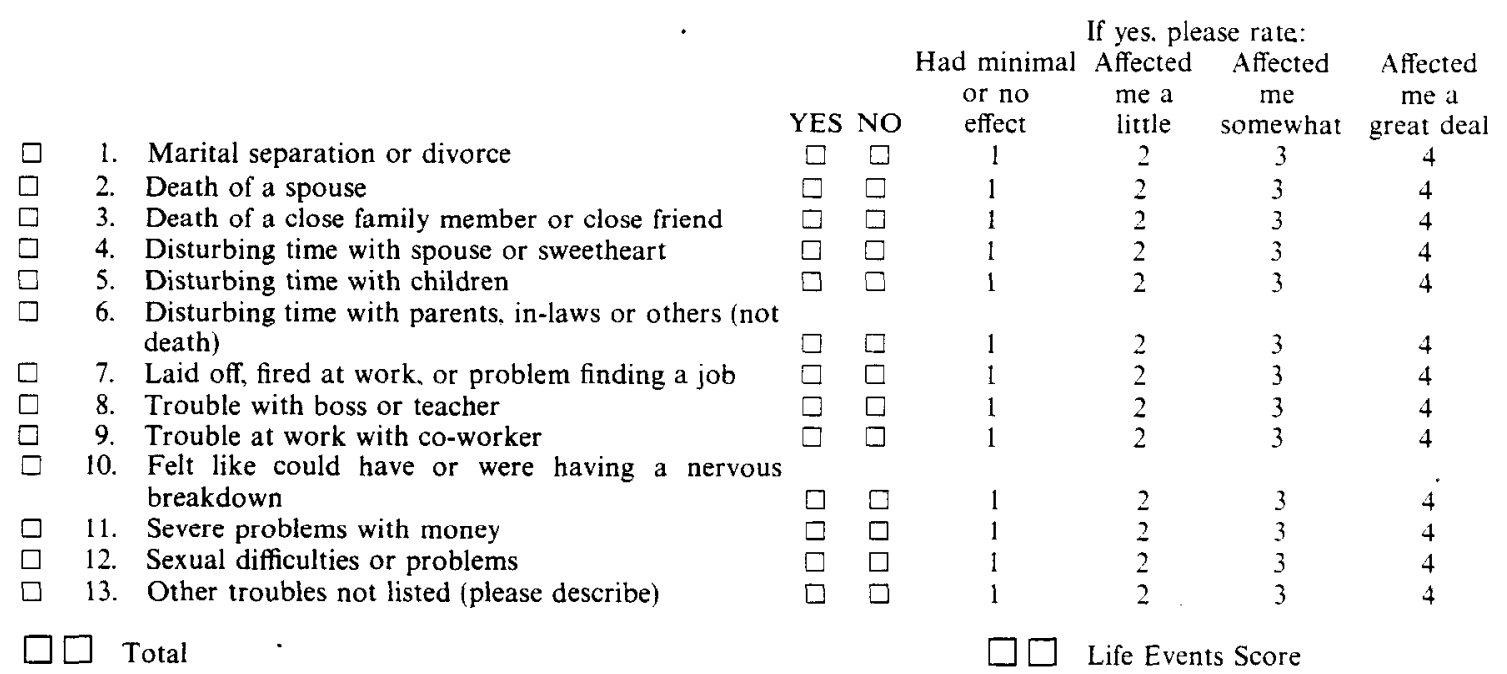

Fig. 2. This is a modification of the social readjustment rating scale of Holmes and Rahe by E. Harburg. The mean life events score for the 150 patients studied was $5.0 \pm 5.4$ (mean \pm SD).

(142 $\mathrm{mm} \mathrm{Hg}$ systolic and $84 \mathrm{~mm} \mathrm{Hg}$ diastolic) was significantly lower by pairwise $T$-test, $(P<0.0001)$ than the initial measurement $(178 \mathrm{~mm} \mathrm{Hg}$ systolic and $108 \mathrm{mmHg}$ diastolic). The number and percent of those in each group and their final blood pressures were as follows: poor control $16(11 \%)$, mean systolic blood pressure $179 \pm 7.3( \pm \mathrm{SE}) \mathrm{mm} \mathrm{Hg}$, mean diastolic blood pressure $106 \pm 2.7 \mathrm{~mm} \mathrm{Hg}$; satisfactory control $52(35 \%)$ mean systolic hlood pressure $153 \pm 1.9 \mathrm{~mm} \mathrm{Hg}$, mean diastolic blood pressure $86 \pm 1.1 \mathrm{mmHg}$; excellent control $82(55 \%)$, mean systolic blood pressure $128 \pm 1.2 \mathrm{~mm} \mathrm{Hg}$, mean dias-

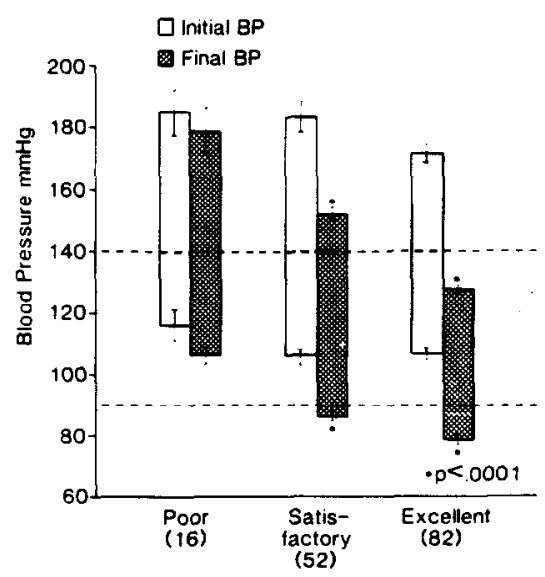

BLOOD PRESSURE CONTROL

Fig. 3. Blood pressure levels by control groups (mean $\pm \mathrm{SE}$ ). Blood pressure responses to antihypertensive treatment for each of the three groups based on the degree of blood pressure control achieved. tolic blood pressure $79 \pm 0.9 \mathrm{~mm} \mathrm{Hg}$. Because the mean diastolic level of final blood pressures for those with satisfactory control and those with excellent control were both below $90 \mathrm{~mm} \mathrm{Hg}$, the two groups could be combined and said to have good control, 134 $(89 \%)$. Only $16(11 \%)$ had poor control. Those with satisfactory control but not less than $140 / 90 \mathrm{~mm} \mathrm{Hg}$ included many older patients who had faintness or weakness when their systolic blood pressures were lowered below 150 or $160 \mathrm{~mm} \mathrm{Hg}$.

\section{Age}

Older persons tended to have better blood pressure control than younger individuals. The $16(11)$ of 150 with poor control had a mean age of $53 \pm 11$ years, the $52(35 \%)$ of 150 with satisfactory control had a mean age of $62 \pm 9$ years reflecting the larger number of these who were judged by the physician to have satisfactory control (although not below $140 / 90 \mathrm{~mm} \mathrm{Hg}$ ) because of their increased age. However, even among those with excellent control $[82$ $(55 \%)$ of 150 who had blood pressures consistently below $140 / 90 \mathrm{~mm} \mathrm{Hg}$, the mean age was greater ( $58 \pm 9$ years) than the mean age of those with poor control. By ANOVA this difference was statistically significant $(P=0.009)$.

\section{Weight}

The patients averaged $35 \pm 32$ (mean \pm SD) pounds above their ideal body weight. Their percent of ideal body weight as a group was $125 \pm 22^{\circ}$ \% Using \% ideal body weight $(\%$ IBW) as an independent variable, least squares regression shows that as the $\%$ IBW increases, the number of antihypertensive tablets increase $(r=0.25, P=0.0021)$. As $\%$ IBW increases, compliance index decreases $(r=-0.23$. $P=0.0045$ ). Thus, increased weight is associated with 
both the number of tablets prescribed and reduced compliance with the regimen.

\section{Severity of hypertensive disease}

There was no statistically significant association between the adequacy of blood prcssure control and cardiac enlargement (roentgenogram). left ventricular hypertrophy by electrocardiogram, azotemia, proteinuria or retinopathy at initial examination. At the final examination. these markers of target organ damage were not significantly associated with blood pressure control, except for proteinuria $\left(\chi^{2}=12.3\right.$, $P<0.02)$.

\section{Health in general}

Despite the fact that severity of hypertensive disease did not correlate with adequacy of blood pressure control, "health in general' did. One of the questions in the interview-questionnaire was-"At the present time, would you say that your health in general is excellent, good, fair, or poor?" Fifty-six (37\%) of 150 answered, 'fair" or 'poor' health and of these 13 $(23 \%)$ of 56 had poor control. Among 90 who rated their health as 'good' or "excellent", only $3(3 \%)$ had poor control (Fig. 4).

\section{Mean number of tablets per day}

For those with poor control, the mean number of antihypertensive tablets prescribed per day was significantly greater than for those with satisfactory and excellent control (Fig. 5). Thus, it is indicated that individuals with poor blood pressure control require more aggressive treatment, which in turn results in a more complicated regimen for them to follow.

\section{Appointments kept}

From the medical record review, it was possible to assess the number of appointments kept during the previous year. Thirty $(20 \%)$ of $150 \mathrm{kept}$ fewer than $75 \%$ of their appointments, and of these. $11(37 \%)$ had poor control vs $5(4 \%)$ of 119 who kept $75 \%$ or more

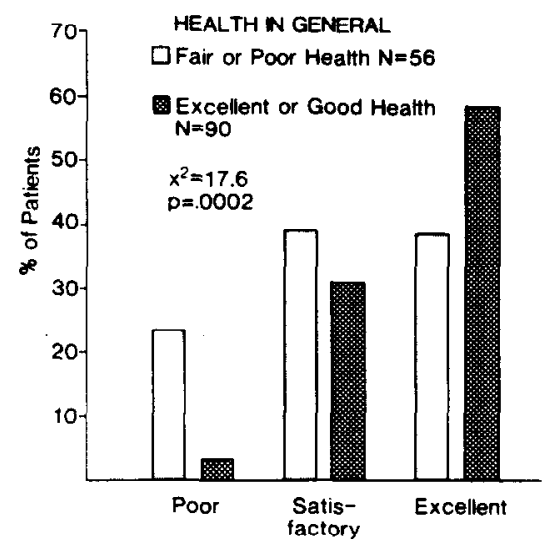

BLOOD PRESSI IRE CONTROL

Fig. 4. Ninety patients rated their health as excellent or good. Of these $90.3\left(3^{\circ}\right)$ had poor. $\left(32^{\circ}\right)$ had satisfactory and $58\left(64^{\circ}{ }_{0}\right)$ had excellent blood pressure control. Excellent or good general health in these patients was usually associated with excellent blood pressure control.

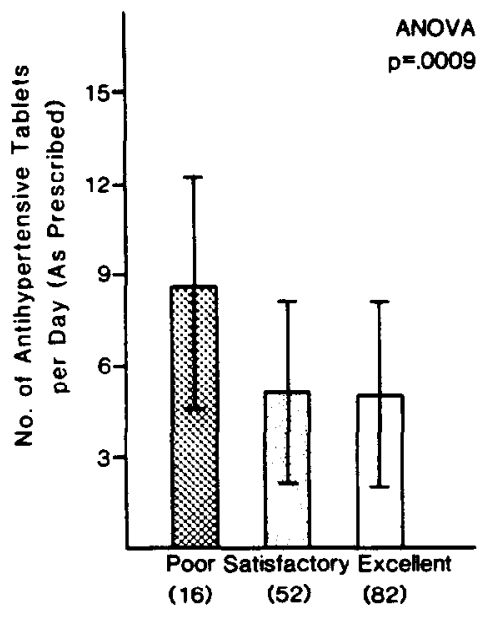

Blood Pressure Control

Fig. 5. Antihypertensive tablets per day: mean $\pm S D$ vs blood pressure control. The number of antihypertensive tablets prescribed per day was significantly greater in those with poor blood pressure control than in the others.

of their scheduled appointments (Fig. 6A). The number of appointments kept proved to be a good indicator of compliance with antihypertensive treatment and was one of the indices used in development of our compliance index.

\section{Marital status}

Thirty-six $(24 \%)$ of the 150 patients were not married. This included those who were widowed, divorced, separated or single. Eleven $(31 \%)$ had poor control vs $5(4 \%)$ of 114 married persons (Fig. 6B).

\section{Work status}

Seven $(33 \%)$ of 21 who were retired for any medical disability had poor blood pressure control (Fig. 6C) Fifteen $(52 \%)$ of 29 retired persons had satisfactory control which reflects the fact that older patients with isolated systolic hypertension (under treatment) were judged to be under satisfactory control even though their systolic blood pressures were not less than $140 \mathrm{~mm} \mathrm{Hg}$. The category entitled 'other, not working' included those who were housewives, unemployed, laid off, and students. Forty-five $(65 \%)$ of the 69 people who were 'working' at the time of the study had excellent control.

Income

Ten $(24 \%)$ of 42 patients with income below $\$ 9000$ a year had poor control. This is in contrast with 3 $(10 \%)$ of 31 with income $\$ 9000-\$ 20,000$ a year and only $2(3 \%)$ of 63 with incomes of $\$ 20,000$ or more a year. Conversely. a progressively higher percentage of people with higher incomes had excellent control. Forty-one $(65 \%)$ of 63 patients with an income of $\$ 20,000$ a year or more had excellent control (Fig. 6D).

\section{Compliance index and blood pressure control}

A histogram (Fig. 7) of the compliance index stratjfied by the adequacy of blood pressure control shows 
that patients with excellent blood pressure control had the highest compliance index. As the adequacy of blood pressure control improved, the compliance index increased and the variance of the index between the groups decreased. There is a statistically significant difference ( $P<0.0001)$ between the lower compliance index of those with poor blood pressure con-

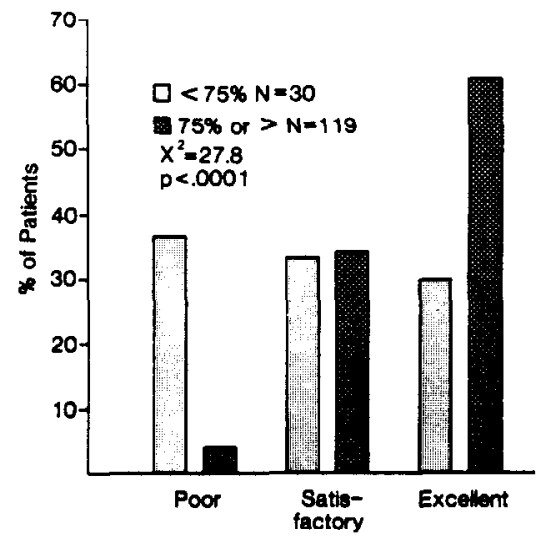

BLOOD PRESSURE CONTROL

(A)

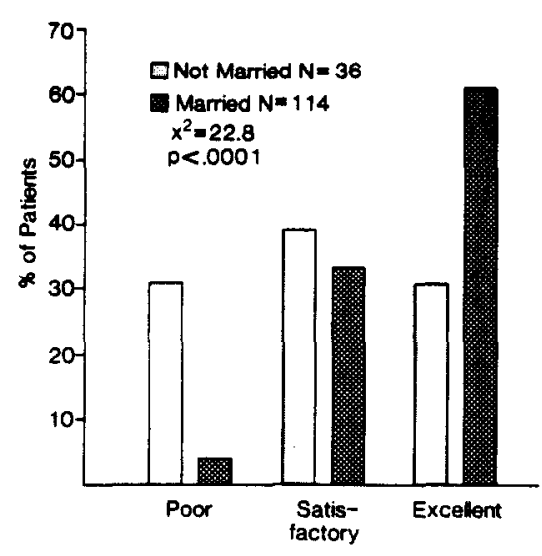

BLOOD PRESSURE CONTROL.

(B)

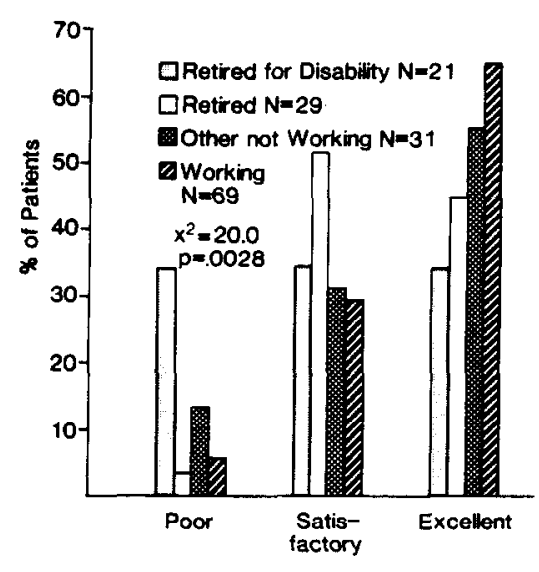

BLOOD PRESSURE CONTROL

(C)

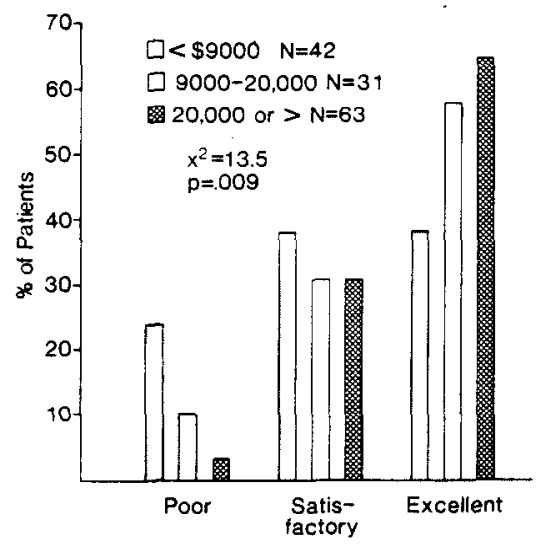

BLOOD PRESSURE CONTROL

(D)

Fig. 6. Behavioral and socioeconomic variables which were significantly related to the degree of blood pressure control: (A) appointments kept; (B) marital status; (C) work status; (D) income group.

trol and the higher compliance index in those with good blood pressure control.

A scatter plot and linear regression relating systolic blood pressure to compliance index shows a negative relationship: as compliance index increases, systolic blood pressure falls $(r=-0.26, P=0.0016)$. Similarly, diastolic pressure falls as compliance index increases $(r=-0.35, P<0.0001)$ (Fig. 8A.B).

\section{Mean life events score}

During the interview, the patient was asked, "How many of these life events did you experience within the past six months?" In addition to the number of life change units, a measure of the degree of impact on the individual was obtained through the interview. The total scores ranged from 0 to 16 . For patients with poor blood pressure control, the mean score was $9 \pm 7$ (SD), with satisfactory control $4.3 \pm 5$ and for

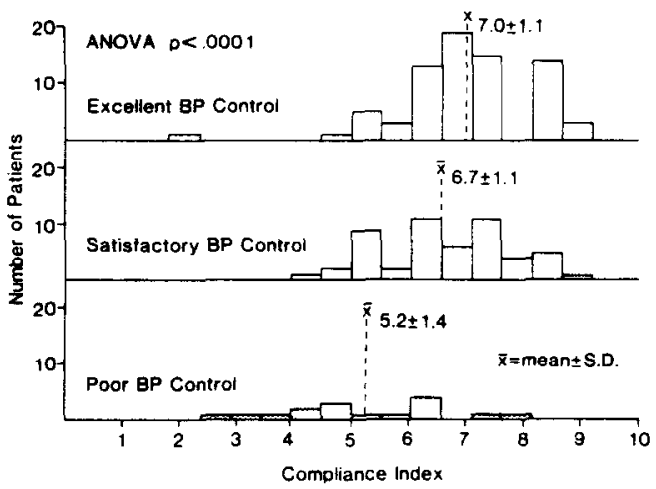

Fig. 7. Blood pressure control vs compliance index. Histogram of compliance index for each of three groups of patients grouped according to degree of blood pressure control. As the adequacy of biood pressure control improved. the compliance index increased and the variance of the index in the groups decreased. 

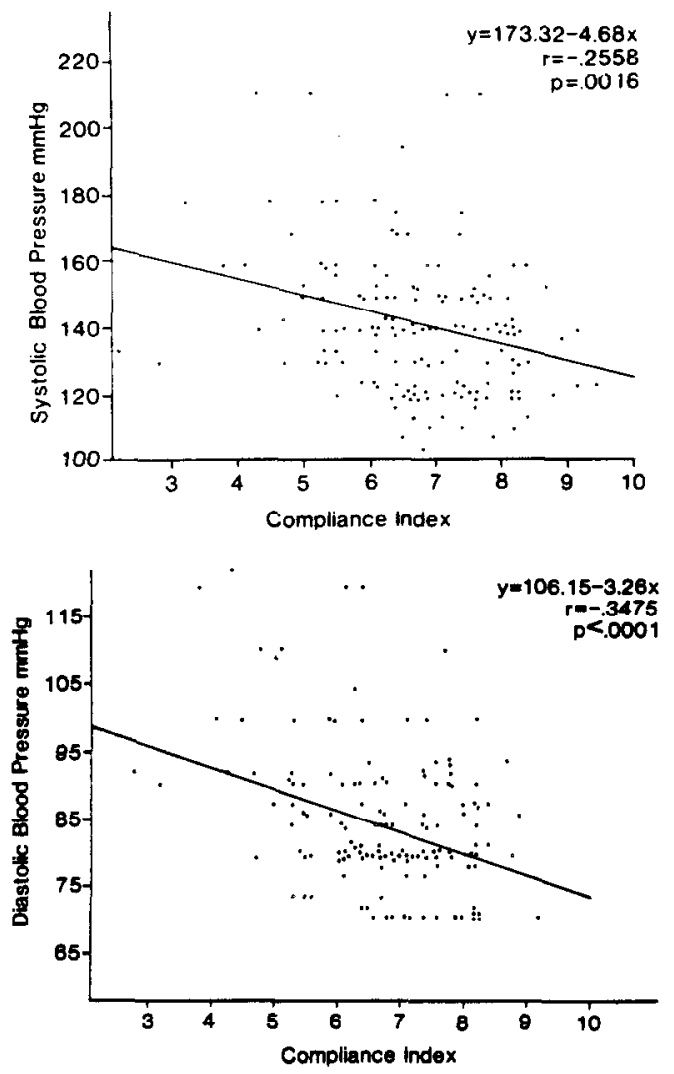

Fig. 8. Scatter plots for systolic blood pressure vs compliance index and for diastolic blood pressure vs compliance index. As compliance index increases, systolic blood pressure falls. Also, as compliance index increases diastolic blood pressure falls.

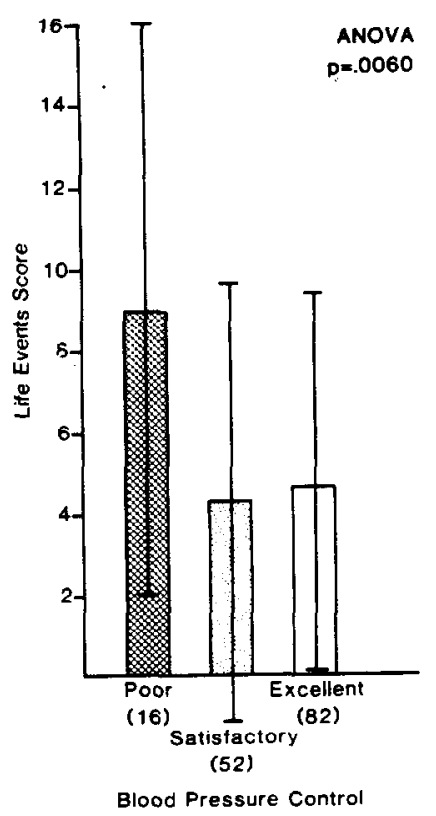

Fig. 9. Mean life events score (with SD) vs blood pressure control. Life events scores were determined from the responses to the questionnaire in Fig. 2. Although patients with poor blood pressure control had significantly higher scores. the standard deviations are very large.
Table 3. Statistics for the compliance index and other factors influencing blood pressure control

\begin{tabular}{lll}
\hline \multicolumn{1}{c}{ Factor } & Statistics & Significance \\
\hline Age & $r=0.23$ & $P=0.0042$ \\
\% Ideal body weight & $r=-0.23$ & $P=0.0045$ \\
No. of antihypertensive & & \\
$\quad$ tablets per day & $r=-0.24$ & $P=0.0028$ \\
Life cvents scorc & $r=-0.25$ & $P=0.0018$ \\
Appointments kept & $\chi^{2}=113.5$ & $P<0.0001$ \\
Complexity of stepped & & \\
$\quad$ care regimen & $\chi^{2}=36.1$ & $P<0.001$ \\
Marital status & $\chi^{2}=47.0$ & $P<0.014$ \\
Income & $\chi^{2}=85.7$ & $P=0.0065$ \\
Health in general & $\chi^{2}=29.6$ & $P=0.38$ \\
Work status & $\chi^{2}=26.6$ & $P=0.97$ \\
\hline
\end{tabular}

excellent control $4.7 \pm 5$. By ANOVA this difference is significant $(P<0.006)$. However, there is a very large standard deviation of the life events scores in all groups (Fig. 9).

\section{Relation between compliance index and other factors}

There was a significant association between the compliance index and other factors influencing blood pressure control. These included age, percent of ideal body weight, number of antihypertensive tablets per day, life events score, percentage of medical appointments kept during the past year, complexity of stepped care regimen, marital status and income. All of these variables were significant at less than $P=0.05$ (Table 3). Health in general and work status did not show a statistically significant relation to the compliance index.

\section{DISCUSSION}

The Joint National Committee on Detection, Evaluation and Treatment of High Blood Pressure has emphasized that certain factors are necessary to achieve good blood pressure control. These include a good physician-patient relationship, aggressiveness of treatment by the physician, an appropriate stepped care regimen to achieve goal blood pressure and a careful consideration of the side effects of drugs in tailoring the regimen to the patient's needs [11]. Compliance is also necessary to achieve good blood pressure control. Poor compliance is the outcome of a complex interaction between the patient, the physician, the disease process, the treatment regimen and the therapeutic milieu [12]. Altering the last by such a simple measure as sending out notices of missed appointments can improve compliance [13].

In our study, however, factors in the patients' lives emerged as significant influences on the adequacy of blood pressure control. We have identified psychosocial and socioeconomic, as well as physical factors, which are associated with the degree of blood pressure control in moderate and severe hypertension. A statistically significant association has been shown for eleven variables. We have assessed compliance by the medical interview-questionnaire and chart review. The interview has been proven. by a number of investigators. to be preferred over other methods of com- 
pliance assessment such as pill counts $[14,15]$. In a previous study which was also based on interviewquestionnaire and chart reviews, the more rigorously the patient adhered to antihypertensive regimens. the lower the blood pressure. Also, patients who had accurate knowledge of their regimens and those who perceived the consequences of non-adherence as serious, were likely to have lower levels of blood pressure [16].

Fletcher and co-workers have shown that there is a set of medical care processes and patient characteristics which discriminate between groups of well controlled and uncontrolled hypertensive patients and which predicts blood pressure outcome. They also found that older patients achieve better blood pressure control than younger ones [17]. We confirm the latter finding. Younger individuals are also less likely to have had traumatic-morbid events due to their disease. Such events reinforce compliance [8].

It is not surprising that patients who weigh more need more tablets to keep blood pressure under control. In our experience, many patients can achieve a striking reduction in the number of antihypertensive tablets required as they reduce their weight to normal. The reduction in compliance index as \% IBW increases (see Table 3 ) could also reflect psychological problems in these patients.

Other authors have noted that health in general, and particularly the patient's perception of his health status. are factors in blood pressure control $[18,19]$ Disability retirement associated with poor blood pressure control reflects the morbidity or medical consequences of severe hypertensive disease. In this instance. the intractibility of the hypertensive disease adversely affects the socioeconomic status of the patient rather than the reverse.

Keeping all scheduled appointments and taking a large number of tablets present obstacles to compliance with the treatment prescribed. Numerous investigators have studied various aspects of the drug regimen [20-25]. The demands of the regimen may be too much for the patient to follow [16], especially if he has poor coping ability as a result of his disease [26]. The high cost of medical care is an additional burden on the economically deprived patient. Fully, one third of patients who had an emergency after dropping out of treatment, cited insufficient funds as the reason they dropped out of therapy [8].

Married people are more likely to have stronger social support, which is often lacking in the widowed. divorced and separated. The spouse usually encourages the partner to do everything possible to take care of his/her blood pressure. Social support has proven to be important in adherence to medical regimens [16].

\section{Socioeconomic factors and compliance}

Sackett and Haynes have cited many references in support of a significant role of socioeconomic factors in compliance, as well as a large number of references which show no such relationships $[27,28]$. In an earlier study, patients who had experienced a hypertensive emergency following discontinuance of therapy were compared to a group of compliant patients. The dropouts were younger, had the disease for a shorter time, had less education, less income and were most often black [8]. Other studies also show a relationship between low socioeconomic status and compliance [29-31]. However, when Haynes et al. [14] studied 145 hypertensive, middle-aged male steel workers who were treated primarily for mild hypertension ( $78 \%$ were on one medication alone) by many different physicians, $43 \%$ did not achieve good blood pressure control. Their study did not show a significant association between socioeconomic variables and compliance. By contrast, in our study, 150 patients of diverse age, sex, race, occupational status and income were included. All of our patients had moderate or severe, rather than mild, hypertension and required at least two different types of antihypertensive drugs. Also, all were treated by the same physician and $89 \%$ achieved good blood pressure control. Our results confirm a positive relationship between socioeconomic factors and compliance, in patients with moderate and severe hypertension. The Hypertension Detection and Follow-Up Program planners recognized some of these factors when they provided free medical care, free transportation and maximally supportive medical service to ensure good adherence to treatment for their stepped care patients [1].

\section{The compliance index}

The compliance index includes five components of compliance that are of well established value [32-34]. We aimed to develop a useful index of compliance that could be applied readily to any patient who has remained under continuing treatment for hypertension for 1 year or more. Compliance with every aspect of the regimen is important and different aspects are interdependent. Sackett and Haynes make the important observation that improved compliance is associated with a consistent therapist, patient satisfaction, increased supervision and increased regimen simplicity [35]. In this study, we have met all of these conditions. All patients were under direct care of the senior author and most of the patients indicated satisfaction with treatment. Increased supervision was provided for suspected noncompliant patients by referral to our clinical nurse specialist, especially those who were on complex drug regimens and those with difficult life situations. Patients were seen by the nurse at one visit and the physician at the next visit so that continuity of care with both the physician and the nurse was maintained. Since many of these patients had severe hypertension. it was impossible to achieve goal blood pressure levels without complex regimens. Even so, appointments were scheduled at convenient hours when possible, and medications were prescribed in twice daily doses when possible. Aggressive changes in medication were made to achieve the goal with the minimum of side effects. Patients with poor control received more aggressive stepped care regimens and a greater number of prescribed antihypertensive tablets daily as evidence that they were not undertreated. If the physician is not aggressive in the management of hypertension or the patient is not compliant, good blood pressure control cannot be achieved.

Our compliance index was correlated significantly with the adequacy of blood pressure control. This supports the assertion [5-7] that noncompliance is the major reason for inadequate blood pressure con- 
trol. We believe that the compliance index may be a useful tool in clinical research and practice.

\section{Life events score}

The life events score raises the possibility that disturbing life situations may affect blood pressure control. The patient must make the decision to control his blood pressure, take medication as prescribed, monitor progress toward his blood pressure goal and resolve problems that impede good blood pressure control [36]. Disturbing life events may affect patient behavior that is critical to blood pressure control. Hypertension, which is difficult to control, imposes demands on the patient which may be too great to meet, if competing life problems exist [16].

\section{CONCLUSION}

The relationship between the compliance index and other factors studied, appears to offer an answer to the question we originally asked: why are some intensively treated hypertensive patients inadequately controlled? Some patients have inadequate blood pressure control because psychological and socioeconomic factors affect compliance with antihypertensive regimens. These determinants of compliance may also operate in mild hypertension. Psychosocial and socioeconomic factors may be useful in predicting which patients are most likely to suffer inadequate blood pressure control.

Acknowledgements-This research was supported by the Biomedical Research Support Grant to Henry Ford Hospital from N.I.H. SD7-RR-05490, and grants from Mrs Doris Boschan and Mrs Elsa Oppenheim.

The authors also acknowledge Ernest Harburg. School of Public Health. The University of Michigan, Ann Arbor, Michigan as consultant.

We thank John M. Caldwell. Mt Pleasant, Michigan and Max V. Wisgerhof and Oscar A. Carretero of the Henry Ford Hospital for helpful suggestions in revising the manuscript. The secretarial assistance of Carol Crane is also gratefully acknowledged.

\section{REFERENCES}

1. Hypertension Detection and Follow-Up Program Cooperative Group. Five-year findings of the hypertension detection and follow-up program-1. Reduction in mortality of persons with high blood pressure. including mild hypertension. J. Am. med. Ass. 242. 2562. 1979

2. Hypertension Detection and Follow-Up Program Cooperative Group. The hypertension detection and follow-up program. Prev. Med. 5, 207. 1976.

3. Berksorn D. M., Brown M. C.. Stanton H., Masterson J.. Shireman L.. Ausbrook D. K.. Mikes D.. Whipple I. T. and Muriel H. H. Changing trends in hypertension detection and control: the Chicago Experience. Am. J. publ. Hlth 70, 389, 1980.

4. Ward G. W. Changing trends in control of hypertension. Publ. Hlth Rep. 93. 31. 1978.

5. Gifford R. W. and Tarazi R. C. Resistant hypertension: diagnosis and management. Amm. intern. Med. 88. 661. 1978.

6. Tarazi R. C. Long-term effective antihypertensive therapy. Ann. intern. Med. 93, 771, 1980

7. Podel R. N. and Gary L. R. Compliance: a problem in medical management. Am. Fam. Physn 13. 74. 1976.
8. Caldwell J. R., Cobb S., Dowling M. and DeJongh D. The dropout problem in antihypertensive treatment: a pilot study of social and emotional factors influencing a patient's ability to follow antihypertensive treatment. J. chron. Dis. 22. 579, 1970.

9. Mitchell H. C. Graham R. M. and Pettinger W. A. Renal function during long-term treatment of hypertension with minoxidil. Ann. intern. Med. 93, 676, 1980.

10. Holmes T. H. and Rahe R. H. The social readjustment rating scale. J. Psychosomat. Res. 11, 213, 1967.

11. Report of the Joint National Committee on Detection. Evaluation and Treatment of High Blood Pressure. A cooperative study. J. Am. med. Ass. 237, 255, 1977.

12. Blackwell B. Counselling and compliance. Patient Couns. Hith Educ. 1, 45, 1978.

13. Takala J., Niemela N., Rosti J. and Sievers K. Improving compliance with therapeutic regimens in hypertensive patients in a community health center. Circulation 59. 540,1979

14. Haynes R. B., Taylor D. W., Sackett D. L., Gibson E. S.. Bernholz C. D. and Mukherjee J. Can simple clinical measurements detect patient noncompliance? Hypertension 2, 757, 1980.

15. Fletcher S. W.. Pappins E. M. and Harper S. J. Measurement of medication compliance in a clinical setting. Comparison of three methods in patients prescribed Digoxin. Archs intern. Med. 139, 635, 1979.

16. Caplan R. D., Robinson S. A. R., French J. R. P. Jr Caldwell J. R. and Shinn M. Adhering to medical regimens: Pilot experiments in Patient Education and Social Support. Ann Arbor Institute for Social Research. University of Michigan, 1977.

17. Fletcher S. W., Deliakis J., Schoch W. A. and Shapiro S. H. Predicting blood pressure control in hypertensive patients: an approach to quality-of-care assessment. Med. Care 17, 285, 1979.

18. Baile W. F. and Gross R. J. Hypertension: psychosomatic and behavioral aspects. Primary Care 6, 167, 1979.

19. Becker M. H. The health helief model and personal health behavior. Hlth Educ. Monogr. 2, 328, 1974.

20. Fischer R. G. Frequency of drug administration: a prime factor in patient compliance and health care cost. J. Miss. St. med. Ass. 19, 161, 1978.

21. Fischer R. G. Compliance-oriented prescribing: simplifying drug regimens. J. Fam. Pract. 10. 427. 1980.

22. Marston $M$. V. Compliance with medical regimens: a review of the literature. Nurs. Res. 19, 312, 1970.

23. Taylor D. W.. Sackett D. L., Haynes R. B., Johnson A. L., Gibson E. S. and Roberts R. S. Compliance with antihypertensive drug therapy. Ann. N.Y. Acad. Sci. 304, 390, 1978.

24. Syme S. L. Drug treatment of mild hypertension: social and psychological considerations. Ann. N.Y. Acad. Sci. 304. $99,1978$.

25. Ryan C. and Wilson A. Controversies in the treatment of hypertension. Compr. Ther. 6, 65, 1980.

26. Shapiro A. P., Miller R. E., King H. E., Guicherean E. and Fitzgibbon $\mathrm{K}$. Behavioral consequences of mild hypertension. Abstr. Circ. 62. Suppl II. Oct. 1980.

27. Sackett D. L. Introduction and the magnitude, determinants and measurements of compliance. In Compliance with Therapeutic Regimens (Edited by Sackett D. L. and Haynes R. B.). Johns Hopkins University Press, Baltimore, 1976.

28. Haynes R. B. A critical review of the "Determinants" of patient compliance with therapeutic regimens. Ibid.

29. Maddock R. K. Patient cooperation in taking medicines: A study involving isoniazid and aminosalicylic acid. J. Am. med. Ass. 199, 169. 1967

30. Neeley E. and Patrick M. L. Problems of aged persons taking medications at home. Nurs. Res. 17, 52. 1968. 
31. Davis M. S. Variations in patients compliance with doctors' advice: an empirical analysis of patterns of communication. Am. J. publ. Hith 58. 274. 1968.

32. Rudd P. and Marton K. I. Non-traditional problems of antihypertensive management. West. J. Med. 131, 179. 1979.

33. Sackett D. L. Hypertension in the real world: public reaction, physician response and patient compliance. In Hypertension: Physiopathology and Treatment (Edited by Genest J., Koiw E. and Kuchel O.l. McGraw-Hill, New York. 1977.
34. Blackwell B. Treatment adherence in hypertension. 4m. J. Pharm. 148. 75. 1976

35. Sackett D. L.. Taylor D. W.. Haynes R. B.. Johnson A. L.. Gibson E. S. and Roberts R. S. Compliance with therapeutic regimens. In Mild Hypertension: Natural History and Management (Edited by Gross F. and Strasser T.). Year Book Medical Publishers. Chicago. 1979.

36. Working group to define critical patient behaviors in high blood pressure control. Patient behavior for blood pressure control. guidelines for professionals. $J$. Am. med. Ass. 241. 2534. 1979. 